\title{
A mixed methods study of the policy and practice of private patient care in acute NHS trusts in England
}

\author{
Author: Sarah Walpole
}

\section{Background}

Between 2012/13 and 2015/16 there was a $12 \%$ real terms increase in NHS trusts' earnings from private patients (PPs). ${ }^{1}$ Approximately $1 \%$ of NHS beds are set aside for PP occupancy. ${ }^{2}$ Little is known about the impacts of NHS trusts' policies and practices in relation to PP treatment.

The aim of this study was to explore NHS trusts' management of PPs and health professionals' experiences, knowledge and perceptions regarding NHS PPs.

\section{Methods}

Documentary analysis of national guidance, ${ }^{3,4}$ local guidelines from three NHS trusts and published market reports ${ }^{1,5}$ informed the development of freedom of information (FOI) requests about NHS PP care. FOI requests were sent to all 153 acute NHS trusts, asking for data on their PP activity (including income and expenditure) and procedures for calculating tariffs for PPs.

An interview pro forma was developed and refined based on feedback from health policy experts. Interviews were carried out with $17 \mathrm{NHS}$ staff members from varied professions, specialties, levels of experience and geographical places of work. Interviews were transcribed and then analysed using thematic analysis.

\section{Results}

Of 153 NHS trusts, 102 responded to the FOI request. Thirtysix gave figures for the expenditure, 23 providing data on administration costs and 13 provided data on capital costs associated with PP treatment. Most trusts responding did not hold the information or could not isolate expenditure on PP treatment from expenditure on NHS patients; a minority stated that they were not willing to release the data due to commercial sensitivity.

Of 78 trusts who gave information about their approach to tariff setting for PPs, $29 \%$ calculate the cost of providing the treatment, compared to $10 \%$ who agree a tariff with insurance companies, $3 \%$ set a price based on market rates, and $29 \%$ who set prices by adding a mark-up to NHS national tariffs.

Thematic analysis of interviews yielded five themes.

> Impacts on availability of resources: prioritisation of PPs over NHS patients, increased time required to treat PPs and for administration related to PPs and investment in resources for use with PPs.

> Patient safety impacts: potential risks when PPs are prioritised on NHS wards, quality in PPUs and deviation from protocols when treating PPs.

> Impacts on training: PPs providing less training opportunities and consultants leading PP care.

> Recuperation of costs: lack of awareness of clinical staff about PP protocols, lack of awareness of clinical staff of which patients are private, PP office awareness of PP admissions and variable approaches to tariff setting and billing for PPs.

$>$ Direction setting: various opinions of clinical staff about whether PPs should be seen in the NHS, motivations of trusts to permit private practice including retention of skilled consultants and health professionals being obligated to support the treatment of PPs.

\section{Conclusions}

NHS trusts vary widely in the extent to which they permit or encourage PP care and approaches to cost measurement and recuperation. NHS professionals' insights into realities of NHS PP care delivery and related concerns, eg patient safety and resource allocation impacts, are valuable for health policy development and implementation.

\section{References}

1 NHS Digital. Hospital Admitted Patient Care Activity. London: NHS Digital, 2011-2015.

2 LaingBuisson. Private acute medical care. UK market reports, 4th ed, London: LaingBuisson, 2016.

3 Department of Health. A code of conduct for private practice. London: DOH, 2004. www.nhsemployers.org/ /media/Employers/ Documents/Pay \% 20and \% 20reward/DH_085195.pdf [Accessed 28 February 2019].

4 Department of Health. Guidance on NHS patients who wish to pay for additional private care, London: DOH, 2009. www.gov.uk/government/uploads/system/uploads/attachment_data/file/404423/ patients-add-priv-care.pdf [Accessed 28 February 2019].

5 LaingBuisson. Private acute medical care. UK market reports, 3rd ed. London: LaingBuisson, 2014. 